\title{
Limitations of using a cancer registry to identify incident primary intracranial tumours
}

\author{
Carl E Counsell, Donald A Collie, Robert Grant
}

\begin{abstract}
The completeness and accuracy of registration of primary intracranial tumours in the Scottish Cancer Registry was compared with a detailed incidence study performed over a two year period (1989-90). Of 228 patients with any primary intracranial tumour in the incidence study, 124 (54\%) were identified as intracranial tumours in the cancer registry. The registry excluded benign tumours (although this was not consistent) and so the sensitivity of the registry varied with tumour type (84\% for neuroepithelial tumours, $22 \%$ meningeal, $29 \%$ sellar, $0 \%$ cranial nerve). Of the $\mathbf{3 1}$ malignant tumours not found in the registry on our initial search, nine were found to have been included between 1989-90 but using different International Classification of Diseases-9th revision (ICD-9) codes or postcodes, and seven were found registered after 1990. Eleven per cent of cases $(18 / 170)$ identified in the cancer registry were excluded from the incidence study: 11 had evidence of an intracranial tumour before 1989 whereas four definitely did not have an intracranial tumour. The cancer registry therefore significantly underestimated the incidence of all primary intracranial tumours, and of malignant intracranial tumours. Incidence studies must use additional methods to identify all primary tumours. Cancer registries should consider registering all primary intracranial tumours and may improve case ascertainment by screening neuroradiology data.
\end{abstract}

Neurosciences,

Western General

Hospital, Crewe Road,

Edinburgh EH4 2XU,

UK

C E Counsell

D A Collie

R Grant

Correspondence to: Dr Carl Counsell, Department of Clinical Neurosciences, Western General Hospital, Edinburgh EH4 2XU.

Received 18 September 1996 and in revised form 12 February 1997

Accepted 18 February 1997 their incidence may be increasing, and public concerns about the role of electric power cables and cellular phones in their aetiology. ${ }^{1}$ However, there has been little investigation of the completeness of registration of primary intracranial tumours in cancer registries and hence their accuracy in assessing incidence. Intracranial tumours pose a particular problem in terms of registration in that a significant proportion are pathologically benign and yet many cancer registries do not record benign tumours. ${ }^{23}$ We wished to assess the completeness and diagnostic accuracy of data on incident cases of primary intracranial tumours from a regional cancer registry by comparing its data with those from a detailed regional incidence study.

\section{Materials and methods}

The Scottish Cancer Registry routinely collects data on malignant (invasive) primary intracranial tumours and intracranial tumours of uncertain behaviour from hospital discharges, outpatient clinics, pathology laboratories, and death certificates. ${ }^{45}$ We searched the Scottish Cancer Registry for all patients resident in Lothian (defined by postcode) who were registered in 1989 and 1990 with the International Classification of Diseases-9th revision (ICD-9) codes relevant to intracranial tumours (see appendix). The patients identified were compared with those identified in a detailed incidence study of all primary intracranial tumours that was performed in the Lothian region of Scotland between 1989 and 1990. The methods of this study have been reported in detail elsewhere. ${ }^{6}$ The sensitivity of the cancer registry for identifying patients with primary intracranial tumours was calculated by dividing the number of patients identified in the registry who were included in the Lothian incidence study by the total number of patients included in the Lothian study. Given that the cancer registry does not record benign tumours, we also measured the sensitivity for each particular tumour type using the revised World Health Organisation's classification. ${ }^{7}$ We also identified the reason why some patients were included in the cancer registry but excluded from the incidence study, and why some patients from the incidence study had not been found in the registry. We searched the 
registry again using the name and date of birth of any patient in the incidence study who was not found in the first search to see if they were included but in a different time period, or using a different ICD-9 code or postcode.

\section{Results}

Two hundred and twenty eight patients with primary intracranial tumours were identified during the two year incidence study. Only 124 of these $(54 \%)$ were identified in the cancer registry under the appropriate ICD-9 codes over the same time period (table). There were large differences in the sensitivities for specific tumour types which was expected given that benign tumours were not included in the registry (table). Most neuroepithelial tumours are considered malignant and $84 \%$ of these were found in the cancer registry. More of the neuroepithelial tumours that are considered to be of high grade malignancy were registered than those that are considered of lower grade malignancy $(87 \% \mathrm{v} 71 \%, \mathrm{P}=0.09)$. If all malignant tumour types were combined (neuroepithelial, haemangioblastoma, craniopharyngioma, CNS lymphoma, germ cell tumours), $78 \%$ were identified in the cancer registry during the two year period $(107 / 138,95 \%$ confidence interval (95\% CI) $71 \%-85 \%$ ). In comparison, most meningeal and sellar tumours are pathologically benign and so few were found in the registry compared with the incidence study $(22 \%$ and $29 \%$ respectively). Most of those that were registered were coded as being of uncertain behaviour (eight meningiomas, nine pituitary tumours), and four (three meningiomas, one pituitary tumour) had not been biopsied.

One hundred and four patients with primary intracranial tumours were not identified in the cancer registry between 1989 and 1990 . Seventy three of these patients $(70 \%, 95 \%$ CI $61 \%-79 \%$ ) had benign tumour types such as meningiomas, cranial nerve tumours, pituitary tumours, and cystic lesions and therefore were not eligible for inclusion in the registry. Thirty one malignant primary tumours (high grade neuroepithelial 12, low grade neuroepithelial eight, primary CNS lymphoma 10, craniopharyngioma one) were not found using our initial search of the cancer registry. Nine of these

Sensitivity of the Scottish Cancer Registry for indentifying patients with incident primary intracranial tumours

\begin{tabular}{lccc}
\hline Tumour type & $\begin{array}{l}\text { Cases identified in } \\
\text { registry }\end{array}$ & $\begin{array}{l}\text { Cases in incidence } \\
\text { study }\end{array}$ & $\begin{array}{l}\text { Sensitivity of } \\
\text { registry (\%) } \\
(95 \% \text { CI) }\end{array}$ \\
\hline Neuroepithelial & 102 & 122 & $84(77-90)$ \\
High grade & 82 & 94 & $87(79-93)$ \\
Low grade & 20 & 28 & $71(51-89)$ \\
Meningeal & 10 & 45 & $22(11-37)$ \\
Meningioma & 8 & 43 & $19(8-33)$ \\
Haemangioblastoma & 2 & 2 & $100(16-100)$ \\
Sellar & 11 & 38 & $29(15-46)$ \\
Pituitary & 9 & 3 & $26(12-43)$ \\
Craniopharyngioma & 2 & 10 & $67(9-99)$ \\
Cranial nerve & 0 & 10 & $0(0-31)$ \\
Primary CNS lymphoma & 0 & 1 & $0(0-31)$ \\
Germ cell & 1 & 2 & 100 \\
Cystic lesion & 0 & 228 & 0 \\
All primary tumours & 124 & $54(48-61)$ \\
\hline
\end{tabular}

${ }^{\star}$ Glioblastoma, anaplastic astrocytoma, anaplastic oligodendroglioma, medulloblastoma, and pineocytoma.

†Low grade astrocytoma, mixed glioma, oligodendroglioma, and ependymoma. patients were found subsequently in the registry between 1989 and 1990 and had been correctly coded: six lymphomas were coded as haematological malignancies (ICD-9 200202), and three neuroepithelial tumours were missed because of errors in postcodes. Seven other patients were registered in 1991 with appropriate ICD-9 codes, whereas another seven were included in the registry before 1989 but only on the basis of a previous extracranial tumour which was not relevant to their intracranial tumour. Eight patients with malignant tumours were not found in the registry at all, five of whom had biopsy established tumours. If the nine patients who were registered between 1989 and 1990 and were correctly coded were included, the sensitivity of the cancer registry increased to $58 \%$ for all tumours and $84 \%$ for malignant tumours, whereas including the 16 with appropriate ICD-9 codes who were registered from 1989 onwards increased the figures to $61 \%$ and $89 \%$ respectively.

Eighteen out of 170 patients (11\%) found during our initial search of the registry were excluded from the incidence study. Eleven had evidence of an abnormality compatible with a tumour on CT performed before 1989, and three had a spinal tumour (ICD-9 codes 237.5, 237.6). The remaining four patients were excluded because the diagnosis was made on purely clinical grounds and no CT or biopsy had been performed (one patient); the final diagnosis was a stroke (one patient); the patient was a foreign national who was not resident in Lothian (one patient); and the notes, CT, and histology were not available to confirm the diagnosis (one patient).

\section{Discussion}

We have identified several difficulties in using the Scottish Cancer Registry to identify incident primary intracranial tumours which will apply to other registries using similar methods. Similar problems have been shown in the registration of other tumours in other cancer registries. ${ }^{8}$ The registration of primary intracranial tumours was incomplete, mainly because it excluded so called benign tumours. There are problems with only registering malignant tumours. Firstly, benign tumours are common, accounting for about $50 \%$ of all primary intracranial tumours, ${ }^{6}$ and therefore excluding them will significantly underestimate the burden of disease. Secondly, the definition of "malignancy" depends largely on histology, which is not always available (69\% of patients in the cancer registry and in the incidence study had histological confirmation). Thirdly, the histological grading of intracranial tumours is often difficult, ${ }^{9-11}$ and may be susceptible to between and within observer errors. ${ }^{12}$ Finally, the clinical consequences of a "benign" intracranial tumour can be just as serious as a "malignant" one in terms of neurological impairment and prognosis. The 12 regional cancer registries in England and Wales do include benign intracranial tumours but the registration is often incomplete. ${ }^{13}$ For example, in 1989 the incidence of tumours coded as 
ICD 225 (almost all benign meningiomas) ranged from $0.2 / 100000$ in the West Midlands region to $2.0 / 100000$ in the South Western region. $^{13}$

Registration of malignant tumours was also incomplete. Eleven per cent of these were either not included in the registry or were included only because of previous diagnosis of an extracranial tumour. The reasons why these patients were not included are unclear. Most had biopsies or had a brain tumour included in the death certificate and so should have been detected by the registry. Other problems existed in the registration of malignant tumours. The ICD-9 coding system did not allow intracranial tumours of uncertain behaviour to be differentiated from intraspinal tumours (codes 237.5, 237.6); nor did it identify primary CNS lymphomas as intracranial tumours. The new ICD-10 classification, ${ }^{14}$ which most cancer registries are starting to use, is an improvement as it does differentiate between intraspinal and intracranial tumours of uncertain behaviour. However, primary CNS lymphomas are still not coded as intracranial tumours. One solution to this would be to search registries using the ICD-0 classification instead as this records both the site and morphology of the tumour. ${ }^{14}{ }^{15}$ There were also discrepancies between the Lothian study and the cancer registry in patients' addresses and the date of diagnosis, which meant that some patients were missed by our initial search. The Lothian incidence study used the date of the first abnormal scan (or necropsy in those not scanned) as the date of diagnosis whereas the cancer registry does not use a consistent date which makes it difficult to use for incidence studies. It usually uses the date of biopsy but there are many patients who do not have a histological diagnosis. The difference in the definition of the date of diagnosis meant that 11 patients included in the registry were excluded from the Lothian study because of abnormal scans before 1989 but this was balanced by the fact that seven patients from the Lothian study were registered in the cancer registry after 1990 .

The exclusion of benign tumours from the registry was also not consistently applied. For example, relatively benign low grade gliomas such as juvenile pilocytic astrocytomas and oligodendrogliomas were included as were some pituitary adenomas and meningiomas. A firm diagnosis of malignant pituitary tumours or meningiomas requires histology, and yet $25 \%$ of these tumour types included in the registry did not have a biopsy. There is also a lack of consistency among different cancer registries as not all registries exclude benign tumours. ${ }^{3}$ Despite the problems with completeness and consistency, the diagnostic accuracy of the cancer registry was good. Only six patients $(2 \%)$ identified from the registry were excluded from the Lothian study because the diagnosis of primary intracranial tumour was not confirmed, three of whom had spinal cord tumours.
RECOMMENDATIONS

We would support the recommendation that all (benign and malignant) primary intracranial tumours be included in cancer registries. ${ }^{2}$ This would give a more accurate picture of the total burden of disease for planning health care, would improve the usefulness of the registries for epidemiological studies, and would remove the inconsistencies that presently exist. Complete case ascertainment of any tumour by cancer registries is probably not possible or cost effective. ${ }^{16}$ However, the registration of intracranial tumours could be improved if cancer registries screened neuroradiology reports, which should become easier as more radiology departments computerise their data collection. This would overcome the problem of identifying patients who do not have a biopsy ( $31 \%$ of patients in the Lothian study), who are not admitted to hospital (12\% of patients in the Lothian study), or whose hospital discharge data are miscoded. ${ }^{17}$ However, these improvements would require increased resources at a time when many registries are already underresourced. ${ }^{18}$ Primary CNS lymphomas should also be more easily identified as intracranial tumours.

In summary, the Scottish Cancer Registry did not produce accurate figures for the incidence of benign or malignant primary intracranial tumours, and it is likely that similar problems exist in other cancer registries. Those who wish to perform studies of the incidence and epidemiology of these tumours must, therefore, use other methods, in addition to cancer registries, to maximise case ascertainment. Otherwise, they may only identify about $50 \%$ of all primary tumours and $85 \%$ of malignant tumours. Close collaboration between researchers and the cancer registries will help to improve both future incidence studies and cancer registry data.

We thank the staff of the Scottish Cancer Registry for providing us with the data for this study, particularly Linda Sharp (now at the University of Aberdeen) who also provided helpful comments on the manuscript. We also thank the Information and Statistics Division of the Scottish Health Service, and the General Register Office for providing us with additional data; all clinicians involved in the care of the patients in the Lothian study who helped to identify them, in particular Dr A Gregor (oncology), Mr IR Whittle (neurosurgery), and Dr JW Ironside (neuropathology); and $\mathrm{Mr}$ Stuart Griffen (audit assistant) who (neuropathology); and Mr Stuart Griffen (audit assistant) who helped trace many of the case notes. This project was made possible by a grant from the Lothian Medical Audit Committee. CC was supported by a Wellcom
fellowship in clinical epidemiology.

\section{Appendix: ICD-9 codes of intracranial tumours registered in Scottish Cancer Registry ${ }^{19}$}

191 Malignant neoplasms of brain

192 Malignant neoplasm of other and unspecified parts of nervous system

.0 Cranial nerves

.1 Cerebral meninges

8 Other

.9 Part unspecified

194 Malignant neoplasm of other endocrine glands and related structures

.3 Pituitary and craniopharyngeal duct

.4 Pineal gland

237 Neoplasm of uncertain behaviour of endocrine glands and nervous system

.0 Pituitary and craniopharyngeal duct

.1 Pineal gland 
.5 Brain and spinal cord

.6 Meninges

.9 Cranial nerves

239 Neoplasm of unspecified nature

.6 Brain

.7 Other parts of nervous system (cranial nerves, meninges).

1 Inskip PD, Linet MS, Heineman EF. Etiology of intracranial tumors in adults. Epidemiol Rev 1995;17:382-414.

2 Davis FG, Malinski N, Haenszel W, Chang J, Flannery J, Gershman S, et al . Primary brain tumor incidence rates in four United States regions, 1985-9: a pilot study. Neuroepidemiology 1996;15:103-12.

3 Muir CS, Storm HH, Polednak A. Brain and other nervous system tumours. In: Cancer surveys volume 19/20: trends in cancer incidence and mortality. London: Imperial Cancer Research Fund, 1994.

4 Muir CS. Cancer registry in cancer control: an overview with a Scottish dimension. Health Bull 1993;51:208-29.

5 Sharp L, Black RJ, Harkness EF, Finlayson AR, Muir CS. Cancer registration statistics in Scotland. 1981-90. Edinburgh: ISD Publications, 1993.

6 Counsell CE, Collie DA, Grant R. The incidence of intracranial tumours in the Lothian region of Scotland, 1989-90. f Neurol Neurosurg Psychiatry 1996;61:143-50.

F Neurol Neurosurg Psychiatry 1996;61:143-50.
7 Kleihues P, Burger PC, Scheithauer BW, in collaboration Kleihues P, Burger PC, Scheithauer BW, in collaboration
with LH Sobin and pathologists in 14 countries. World Health Organisation international histological classification of Health Organisation international histological classification of
tumours. Histological typing of tumours of the central nervous tumours. Histological typing of tumours

system. Berlin: Springer-Verlag, 1993 .
8 Nwene U, Smith A. Assessing completeness of cancer registration in the North-Western Region of England, by a method of independent comparison. Br f Cancer 1982;46: $635-9$.
9 Armstrong DA, Almes MJ, Buffler P, Frankowski F, McGarry P. A cluster classification for histologic diagnosis of CNS tumors in an epidemiologic study. Neuroepidemiology 1990;9:2-16.

10 Rohringer $M$, Sutherland GR, Louw DF, Sima AAF. Incidence and clinicopathological features of meningioma. f Neurosurg 1989;71:665-72.

11 The Childhood Brain Tumor Consortium. Intraobserver reproducibility in assigning brain tumors to classes in the World Health Organisation diagnostic scheme. $f$ Neurooncol 1989;7:211-24.

12 Weller RA. Grading of brain tumours. The British experience. Neurosurg Rev 1992;15:7-11.

13 Office of Population Censuses and Surveys. Cancer statistics registrations. England and Wales, 1989. London: HMSO, 1994.

14 World Health Organisation. International classification of diseases and related health problems. 10th revision. Vol 1. Geneva: World Health Organisation, 1992.

15 Lutz J-M, Coleman MP. Trends in primary cerebral lymphoma. Br f Cancer 1994;70:716-8.

16 Silcocks PBS, Thornton-Jones H, Skeet RG. Can we achieve $100 \%$ ascertainment in cancer registration? Public Health 1989;103:23-30.

17 Pears J, Alexander V, Alexander GF, Waugh NR. Audit of the quality of hospital discharge data. Health Bull 1992;50: 356-60.

18 Brewster D. Improving the quality of cancer registration data. F R Soc Med 1995;88:268-71.

19 World Health Organisation. International classification of diseases. Manual of the international statistical classification of diseases, injuries, and causes of death. 9th rev. Vol 1. Geneva: WHO, 1977. Sensitivity of the Scottish Cancer Registry for identifying patients with incident primary intracranial tumours. 